\title{
Avaliação de um programa de promoção de habilidades sociais para idosos
}

\author{
Rachel Shimba Carneiro* / Eliane Falcone Falcone**
}

${ }^{*}$ UNISUAM - Centro Universitário Augusto Motta; ${ }^{* *}$ Universidade do Estado do Rio de Janeiro (UERJ)

\begin{abstract}
Evidências na literatura apontam a importância da qualidade nos relacionamentos sociais, mas não fornecem dados suficientes que indiquem como auxiliar os idosos a se tornarem socialmente competentes. O presente estudo pretende avaliar a eficácia de um Programa de Promoção de Habilidades Sociais para Idosos (PHSI). A pesquisa contou com a participação de 40 idosos de uma Universidade da Terceira Idade. O grupo experimental reuniu 20 pessoas com idades que variavam de 62 a 83 anos $(M=69,4 ; D P=6,05)$ e o grupo controle apresentou 20 idosos com idades que variavam de 60 a 74 anos $(M=67,1 ; D P=4,61)$. As habilidades sociais foram avaliadas antes e depois da intervenção com follow-up por meio de uma medida de autorrelato e o desempenho através de jogos de papéis em sete situações sociais. Os resultados apontaram melhora significativa no desempenho social dos participantes do grupo experimental, em relação ao grupo controle, nos contextos envolvendo conflito de interesses. Análises intragrupos também confirmaram uma melhora na capacidade de lidar com situações interpessoais que demandam a afirmação e defesa de direitos. Esses resultados constatam que o Programa criado promoveu habilidades assertivas, previamente apontadas na literatura como deficitárias na população de idosos.
\end{abstract}

Palavras-chave: Idoso, Programa de promoção de habilidades sociais, Desempenho social.

\section{Introdução}

O reconhecimento do rápido aumento do número de pessoas idosas, nas últimas décadas, ocasionou um aumento considerável nas publicações de trabalhos científicos sobre o envelhecimento (Baltes, 1995). Tais estudos têm mostrado que o envelhecer não precisa necessariamente ser acompanhado de perdas, nem de doenças ou afastamento social (Capitanini, 2000). Conforme propõem Joia, Ruiz e Donalisio (2007), com o aumento geral da sobrevida da população ressaltam-se a importância de garantir aos idosos não apenas maior longevidade, mas qualidade de vida, bem-estar subjetivo e satisfação pessoal.

A partir de uma revisão de estudos, Braz e Del Prette (2011) apontam as diferentes formas de violência contra os idosos, dentre as quais se incluem a exploração financeira, os mal tratos físicos e psicológicos, bem como a negligência e o abandono. É importante considerar que a ausência de convívio social causa severos efeitos negativos na capacidade cognitiva geral (Katz \& Rubin, 2000), além de depressão e estresse (Freire \& Sommerhalder, 2000). A pobreza de relações sociais como um fator de risco à saúde tem sido considerada tão danosa quanto o fumo, a pressão arterial

\section{Financiamento: FAPERJ}

A correspondência relativa a este artigo deverá ser enviada para: Rachel Shimba Carneiro, UNISUAM Centro Universitário Augusto Motta, Av. Paris, 72, Bonsucesso, Rio de Janeiro, RJ, Brasil. E-mail: rachelshimba@yahoo.com.br 
elevada, a obesidade e a ausência de atividade física (Goleman, 2006; Herculano-Houzel, 2007). Tal afirmativa indica que a deterioração da saúde pode ser causada não somente por um desgaste natural do organismo, sedentarismo ou uso de tabaco, mas, também, pela redução da qualidade das relações sociais (Ramos, 2002). Por outro lado, as relações sociais podem, de várias formas, promover melhores condições de saúde. Capitanini (2000) e Ramos (2007), por exemplo, ressaltam a importância dos relacionamentos sociais para o bem-estar físico e mental e para qualidade de vida na velhice. Diante de todas essas considerações, pode-se perceber que as relações sociais são fontes protetoras e mantenedoras de saúde (Matsukura, Marturano, \& Oishi, 2002) e de grande importância em todos os momentos da vida (Resende, Bones, Souza, \& Guimarães, 2006).

Os comportamentos sociais que levam à realização pessoal, manutenção ou melhora da auto-estima, aumento na qualidade das relações, bem como respeito e ampliação dos direitos humanos básicos são aqueles que contribuem para a competência social e que, por essa razão, são chamados de habilidades sociais (Del Prette \& Del Prette, 2011). O conjunto das habilidades requeridas para relações interpessoais satisfatórias inclui diferentes classes e subclasses dentre as quais as habilidades de comunicação, assertivas, empáticas, de civilidade, de expressividade emocional, de trabalho etc. (Del Prette \& Del Prette, 2001). Falcone (2001) destaca a importância das habilidades empáticas e de sua vinculação às assertivas para que as interações sociais sejam bem sucedidas. A empatia é entendida como a capacidade de compreender e de expressar compreensão acurada sobre a perspectiva e sentimentos de outra pessoa, além de experimentar compaixão e interesse pelo bem-estar desta (Barret-Lennard, 1993). Já a assertividade é a capacidade de obter satisfação pessoal, por meio da expressão de sentimentos e da defesa dos próprios direitos (Falcone \& Ramos, 2005), possibilitando bem-estar emocional e aumentando a probabilidade da manutenção de relacionamentos interpessoais saudáveis e duradouros (Vila, Gongora, \& Silveira, 2003).

A crescente realização de pesquisas sobre esse tema tem mostrado que as habilidades sociais são comportamentos aprendidos (Del Prette \& Del Prette, 2011) e que podem melhorar a partir de experiências de aprendizagens adequadas (García-Vera, Sanz, \& Gil, 1998). Além disso, não existe nenhuma diferença marcante entre pessoas idosas ou jovens em aprender habilidades sociais (Del Prette \& Del Prette, 2008; Isquick, 1981). Um importante estudo que apontou a aprendizagem das habilidades sociais na terceira idade foi desenvolvido por Isquick (1981), o qual constatou que indivíduos idosos podem mudar padrões de comportamentos usados ao longo da vida e podem aprender novas formas de se relacionar. Braz e Del Prette (2011) avaliaram um programa de habilidades sociais assertivas para idosos, através de associação de método vivencial e técnicas cognitivo-comportamentais, realizado em 10 sessões, o qual revelou melhora significativa dos participantes no escore geral de habilidades sociais, bem como nos escores de autoafirmação na expressão de sentimentos positivos e de habilidades de enfrentamento e autoafirmação com risco. Neri (2004) cita no campo das relações sociais o treino de habilidades sociais como uma das formas em que a psicologia pode contribuir para o bem-estar objetivo e subjetivo dos idosos.

Entretanto, poucos são os estudos que se dedicam a desenvolver habilidades sociais em idosos. A partir de uma ampla revisão de artigos sobre o tema em bases de dados nacionais e internacionais, foram identificados poucos trabalhos que investigaram como desenvolver as habilidades sociais na terceira idade. Para Isquick (1981), isso se deve a uma descrença, por parte dos profissionais de saúde mental, de que os idosos possam fazer mudanças significativas no seu comportamento ou na sua personalidade. Entretanto, o autor sustenta que essa crença é infundada quando métodos apropriados são usados.

Em síntese, a aquisição de um repertório de habilidades sociais através de um programa de intervenção apropriado pode melhorar a saúde e a qualidade de vida dos idosos para o aumento de capacidades sociais e interpessoais para lidar com conflitos de interesses, defesas dos próprios 
direitos, bem como expressão adequada de sentimentos e necessidades. O presente estudo tem como objetivo analisar a eficácia de um Programa de Habilidades Sociais no aumento do repertório de habilidades sociais com idosos.

\section{Método}

\section{Participantes}

Foram selecionados para a pesquisa, 53 idosos que participam de atividades em uma universidade da terceira idade, localizada na cidade do Rio de Janeiro. O recrutamento dessas pessoas foi feito através de cartazes que convidavam aqueles que desejassem: (1) aumentar a capacidade de resolver conflitos interpessoais, reduzindo rompimentos e tornando as relações mais gratificantes, (2) desenvolver habilidades em ouvir e expressar compreensão de forma apropriada e (3) defender os próprios direitos e expressar pensamentos, sentimentos e crenças de forma honesta, direta e apropriada, sem violar os direitos da outra pessoa.

Das 72 pessoas inscritas, 61 compareceram à entrevista que teve como objetivo selecionar os idosos que fariam parte da pesquisa. Dos 61 idosos entrevistados, 53 foram selecionados de acordo com critérios que serão apresentados posteriormente. Um grupo de 26 pessoas foi designado para constituir o grupo experimental e um grupo de 27 participantes constituiu o grupo controle. É importante ressaltar que a distribuição dessa amostra foi baseada na disponibilidade de horários, e que a escolha dos estudantes que fariam parte do grupo experimental e do grupo controle foi baseada na compatibilidade entre a disponibilidade de horário deles e o horário escolhido para o Programa de Habilidades Sociais para Idosos (PHSI). Diante deste fato, que nem todos os componentes da pesquisa tinham disponibilidade de tempo para participar do PHSI, não foi possível fazer uma distribuição aleatória dos idosos entre os grupos controle e experimental.

Do grupo experimental, 2 participantes do sexo feminino desistiram antes de iniciar o PHSI; um participante do sexo feminino compareceu somente ao primeiro encontro; um participante do sexo masculino desistiu após o terceiro encontro e 2 participantes do sexo feminino desistiram por motivos de saúde. Em relação ao grupo controle, 7 participantes não compareceram ao encontro de avaliação na fase pós-treinamento. Por fím, o experimento contou com 20 participantes no grupo experimental ( 1 do sexo masculino e 19 do sexo feminino) e 20 participantes no grupo controle (1 do sexo masculino e 19 do sexo feminino).

Quanto aos dados sociodemográficos, foram investigados a idade, a escolaridade e o estado civil das 40 pessoas que participaram do estudo. O grupo experimental reuniu 20 pessoas com idades que variavam de 62 a 83 anos $(M=69,4 ; D P=6,05)$ e o grupo controle apresentou 20 idosos com idades que variavam de 60 a 74 anos $(M=67,1 ; D P=4,61)$. É importante mencionar que não foi verificada diferença significativa na média das idades entre os grupos experimental e controle $(p<0,001)$.

No grupo experimental, 5 participantes cursaram até o primeiro grau; 5 , até o segundo grau e 10, completaram o curso superior. Com relação ao estado civil, os viúvos predominaram nesse grupo, com 7 pessoas, seguidos por 5 pessoas casadas, 3 divorciadas, 2 separadas e 3 solteiras. Além desses dados, foi verificado que 14 pessoas do grupo experimental têm até 3 anos de tempo de ingresso na Universidade e as outras 6 pessoas têm mais de 3 anos.

Em relação ao grupo controle, verificou-se, ainda, que 3 participantes cursaram até o primeiro grau; 7, até o segundo grau e 10 pessoas completaram o curso superior. Dos 20 participantes, 3 são viúvos; 7 casados; 5 divorciados; 1 separado e 5 solteiros. Por fim, constatou-se que 17 pessoas deste grupo frequentam há no máximo três anos a Universidade e as outras 3 pessoas têm mais de 3 anos de Universidade. 


\section{Instrumentos}

Todos os participantes da presente pesquisa leram e assinaram o Termo de Consentimento Livre e Esclarecido (TCLE). O TCLE teve como objetivo fornecer esclarecimentos sobre a pesquisa, sobre o processo de avaliação dos participantes, tempo e duração do Programa de Habilidades Sociais. Além disso, foi informado sobre a gravação das verbalizações emitidas em sete situações de jogos de papéis, assim como foi explicado que a identidade dos participantes e as verbalizações gravadas seriam mantidas em sigilo.

Para a seleção dos participantes, foram utilizados os seguintes instrumentos: a Escala de Depressão Geriátrica-15 (Almeida \& Almeida, 1999) e o Questionário de Dificuldades em Situações Sociais (Falcone et al., 2001). Tais instrumentos foram aplicados antes da intervenção como critério de inclusão e exclusão para selecionar a amostra. Foram excluídos os estudantes com sintomas depressivos e a inclusão de indivíduos que apresentaram dificuldades em enfrentar algumas situações sociais.

A Escala de Depressão Geriátrica-15 (Almeida \& Almeida, 1999) contém 15 itens que são respondidos com um sim ou um não e foi utilizada para rastreio de depressão. Esta escala foi validada e adaptada no Brasil por Almeida e Almeida (1999). Na apuração dos resultados, o item tem o valor de 1 ponto, quando for identificada a resposta sim nos itens 2, 3, 4, 6, 8, 9, 10 e 15 e, quando for identificada a resposta não nos itens 1, 5, 7, 11, 12, 13 e 14. Caso contrário, ou seja, quando a resposta do participante for diferente do descrito anteriormente, o item vale zero. A soma total de pontos superior a 5 é sugestiva de depressão (Veras, 2002).

O Questionário de Dificuldades em Situações Sociais (Falcone \& cols., 2001) (A7) foi utilizado para selecionar pessoas com deficiências em habilidades sociais. Os itens desse instrumento foram baseados em um estudo realizado por Falcone e cols. (2001), que envolveu a identificação e a descrição detalhada de habilidades encontradas nas 20 situações sociais citadas a seguir: (1) iniciar conversação; (2) encerrar conversação; (3) fazer pedido sem conflito de interesses; (4) fazer pedido com conflito de interesses; (5) fazer pedido de mudança de comportamento; (6) recusar pedidos; (7) responder a críticas; (8) expressar opiniões pessoais; (9) expressar afeto; (10) fazer elogios; (11) receber elogios; (12) defender os próprios direitos em situações nas quais são oferecidos serviços insatisfatórios; (13) convidar alguém para um encontro; (14) conversar com uma pessoa que está revelando um problema; (15) fazer perguntas; (16) cumprimentar alguém; (17) cobrar dívidas; (18) lidar com pessoas com atitudes grosseiras; (19) expressar sentimentos positivos; (20) expressar sentimentos negativos. O Questionário de Dificuldades em Situações Sociais é composto de 20 itens que são respondidos com um sim ou não. Na apuração dos resultados, a resposta sim teve o valor de 1 ponto. Caso contrário, ou seja, quando a resposta do participante foi não, o item teve o valor de zero. Quanto menor a pontuação no questionário, mais chance o idoso tinha de participar da pesquisa. Com base nesse questionário, foram excluídos os estudantes que apresentaram a soma total de pontos superior a 10. É importante notar que não se dispõe, ainda, de estudos sobre as propriedades psicométricas deste questionário, nem padrões normativos para os resultados por ele produzidos.

Para avaliação das habilidades sociais, foi utilizado o Inventário de Habilidades Sociais (IHS; Del Prette \& Del Prette, 2001). Os estudos com o IHS indicaram uma estrutura composta de cinco fatores: (1) Enfrentamento e auto-afirmação com risco; (2) Auto-afirmação na expressão de sentimentos positivos; (3) Conversação e desenvoltura social; (4) Auto-exposição a desconhecidos e situações novas e (5) Autocontrole da agressividade em situações aversivas (Del Prette \& Del Prette, 2001). Conforme consta no Manual do Inventário de Habilidades Sociais de Del Prette e Del Prette (2001), propriedades psicométricas desse instrumento apresentam índices de validade, fidedignidade e consistência interna satisfatórios. É importante considerar que Carneiro, Falcone, Clark, Del Prette e Del Prette (2007) realizaram algumas alterações no IHS para a sua utilização 
com idosos. Os autores modificaram os itens $2,5,8,9,10,11,13,14,15,17,18,22,29,34$ e 38 sem que houvesse alteração no sentido de suas afirmativas.

As situações de jogos de papéis foram utilizadas para provocar as verbalizações dos participantes em situações de: (1) iniciar conversação; (2) recusar pedido; (3) expressar opinião pessoal; (4) cobrar dívida; (5) defender os próprios direitos em situações nas quais são oferecidos serviços insatisfatórios; (6) lidar com pessoas com atitudes grosseiras e (7) fazer pedido com conflito de interesses. Foram criadas categorias de avaliação do comportamento verbal dos participantes para orientar os juízes na avaliação das respostas dos participantes nas sete situações de jogos de papéis. As categorias são: (1) respostas sociais inadequadas; (2) respostas sociais parcialmente adequadas e (3) respostas sociais adequadas. Na categoria número 1, foram enquadradas as pessoas que recusaram-se a enfrentar a situação e acabaram agindo de forma contrária aos seus objetivos pessoais ou agressiva (enfrentaram a situação de forma hostil ou de modo que comprometeu a qualidade da interação). Na categoria 2 foram enquadradas as respostas das pessoas que enfrentaram a situação, defendendo os próprios interesses de forma objetiva, mas não dando atenção à qualidade da interação, ou seja, não demonstrando claramente se importarem com as necessidades, desejos ou sentimentos da outra pessoa. E, por fim, na categoria 3, foram enquadradas as respostas de enfrentamento_que incluem a defesa dos próprios interesses, desejos, necessidades, após demonstração clara de compreensão e de interesse pelos desejos e necessidades do interlocutor. Além disso, foi elaborado um manual contendo explicações e exemplos de respostas sociais inadequadas, respostas sociais parcialmente adequadas e respostas sociais adequadas para cada uma das sete situações de jogos de papéis.

Os pesquisadores contaram com a colaboração de dois psicólogos que avaliaram, como juízes, as verbalizações emitidas nas sete situações de jogos de papéis em três momentos diferentes: (1) linha de base, (2) pós-treinamento e (3) follow-up. Foi realizado um treinamento prévio com os juízes para esclarecer os critérios definidos no Manual citado acima. No período de avaliação, os juízes foram expressamente proibidos de trocar informações entre si. Outro aspecto diz respeito ao fato dos juízes não saberem a qual grupo os participantes pertenciam e também não era identificado, para os juízes, a etapa pré-intervenção, pós-intervenção ou follow-up, com o objetivo de evitar viés na avaliação do comportamento verbal dos idosos.

A partir deste treinamento, os juízes avaliaram as verbalizações dos participantes da seguinte forma: número 1 para as respostas sociais inadequadas, número 2 para as respostas sociais parcialmente adequadas e número 3 para as respostas sociais adequadas. Essas análises foram utilizadas posteriormente para o cálculo de acordos inter-juizes, ou seja, a pesquisadora computou a porcentagem de acordos e desacordos obtidos entre os dois juízes em cada uma das situações de jogos de papéis nas fases pré-treinamento, pós-treinamento e follow-up.

\section{Procedimentos}

O estudo teve início após aprovação em Comitê de Ética em Pesquisa de acordo com Resolução 466/12 do Conselho Nacional de Saúde e foi realizado em duas fases. Na primeira, a pesquisadora marcou um encontro com cada uma das pessoas que responderam ao recrutamento, a fim de avaliar se estas preenchiam os critérios de inclusão para participarem da pesquisa (níveis baixos ou ausência de depressão, deficiências em habilidades sociais).

A segunda fase da pesquisa envolveu a investigação da eficácia do Programa de Habilidades Sociais para Idosos (PHSI). Nesta fase, o participante foi informado sobre a pesquisa e a gravação das verbalizações emitidas em sete situações de jogos de papéis, assim como foi explicado que a identidade dele e as verbalizações gravadas seriam mantidas em sigilo. Além disso, foi explicitado que por razões referentes à pesquisa, alguns idosos selecionados não participariam do PHSI.

O Inventário de Habilidades Sociais (Del Prette \& Del Prette, 2001) e as sete situações de jogos de papéis foram apresentados aos 20 idosos do grupo experimental e aos 20 do grupo controle em 
três encontros de coleta de dados: (1) pré-intervenção, (2) pós-intervenção e (3) follow-up de 30 dias. Nesses três encontros, os participantes foram encaminhados individualmente para uma sala com o objetivo de preencherem o IHS e responderem às situações de jogos de papéis. Para a gravação das verbalizações dos idosos nas sete situações de jogos de papéis, foi usado um gravador portátil Panasonic minicassette recorder.

Após a avaliação na linha de base, deu-se início ao PHSI, o qual foi realizado em 12 encontros de aproximadamente uma hora e meia de duração, duas vezes por semana. Os encontros foram previamente planejados em três partes: (1) verificação de tarefas de casa; (2) breve exposição e discussão da situação social a ser trabalhada, com fornecimento de um texto explicativo sobre o assunto e orientações sobre como desempenhar de forma adequada; (3) dramatização; (4) avaliação do encontro e (5) atribuição de tarefas de casa para promover a generalização das aquisições desenvolvidas nos encontros. Todos os 20 participantes do grupo experimental foram informados que só poderiam ter no máximo três faltas para fazerem parte da pesquisa.

\section{Análise de dados}

Para a extração dos fatores do IHS, foi utilizada uma análise fatorial exploratória com rotação varimax. Além disso, foram calculados os índices de alfa de Cronbach como índice de consistência interna para cada um dos fatores gerados.

Para a comparação do efeito do PHSI, foi utilizado o teste $t$ pareado, considerando a presença de normalidade nas variáveis dependentes (fatores do IHS). O teste $t$ pareado foi realizado para cada um dos grupos (grupo controle e grupo experimental). Dessa forma, foi possível verificar o efeito do programa com base nos fatores do Inventário de Habilidades Sociais (Del Prette \& Del Prette, 2001) para cada um dos grupos. Para verificar a existência ou não de diferenças entre o grupo experimental e o grupo controle no Inventário de Habilidades Sociais (Del Prette \& Del Prette, 2001), foi realizado o teste $t$ entre as médias dos escores de cada grupo em cada fator.

Em relação às sete situações de jogos de papéis, os pesquisadores contaram com a ajuda de dois psicólogos que avaliaram como juízes as verbalizações dos participantes em três momentos diferentes: (1) linha de base, (2) pós-intervenção e (3) follow-up. Levando-se em conta que as verbalizações emitidas pelos participantes, em cada uma das situações de jogos de papéis nos diferentes momentos, receberam de cada juiz uma única avaliação, foi calculado o índice de concordância entre os dois avaliadores por meio do teste Kappa antes e depois da intervenção. O valor de Kappa variou entre 0,70 e 1,00 sendo que a média do Kappa foi 0,879 o que configura uma alta concordância entre os avaliadores. Com base nesses dados, os juizes discutiram os desacordos encontrados entre eles até chegarem a um consenso. Os dados obtidos a partir deste consenso foram então utilizados na análise estatística dos resultados.

Além disso, por se tratar de uma avaliação com três categorias, foi verificada a diferença na frequência das respostas sociais inadequadas, das respostas sociais parcialmente adequadas e das respostas sociais adequadas, entre o antes e o depois da intervenção, utilizando o teste qui-quadrado de contingência. O teste qui-quadrado também foi usado para verificar a existência de diferenças entre o grupo experimental e o grupo controle. Para avaliação global dos jogos de papéis entre grupo controle e grupo experimental, foi utilizado um teste não paramétrico Wilcoxon. O software utilizado foi o SPSS versão 13.0 e o índice de significância adotado foi de $5 \%$.

\section{Resultados}

Os resultados referentes a avaliação dos efeitos do PHSI a partir do Inventário de Habilidades Sociais (Del Prette \& Del Prette, 2001) serão apresentados a seguir. Inicialmente, foi realizada a avaliação da equivalência entre os grupos controle e experimental na fase pré-intervenção. 
A partir da comparação entre as médias dos grupos controle e experimental nos cinco fatores do IHS, na fase pré-intervenção, foi possível constatar diferenças significativas entre os dois grupos no fator auto-afirmação na expressão de sentimentos positivos $(t=-2,65, g l=38, p=0,01) \mathrm{e}$ no fator autocontrole da agressividade em situações aversivas $(t=3,26, g l=38, p=0,00)$. No fator 2, auto-afirmação na expressão de sentimentos positivos, foi observado que o grupo experimental apresentou uma média mais alta do que a do grupo controle, enquanto que o grupo controle obteve uma média mais elevada no fator referente ao autocontrole da agressividade em situações aversivas. Já a comparação entre as médias dos dois grupos, controle e experimental, depois do PHSI, não revelou diferenças significativas em nenhum dos cinco fatores do IHS.

Para avaliar o desenvolvimento e aperfeiçoamento do repertório de habilidades sociais dos participantes do grupo controle e do grupo experimental, foi investigada a diferença das habilidades sociais entre a linha de base e a fase pós-intervenção nos cinco fatores do IHS. O teste- $t$ pareado indicou, nessa análise intragrupos, não haver diferença significativa, para o grupo controle, em nenhum dos cinco fatores do IHS.

Com relação ao grupo experimental, como se vê na Tabela 1, os valores médios, após o PHSI, foram significativamente mais altos que os da fase pré-intervenção nos seguintes fatores: enfrentamento e auto-afirmação com risco $(p=0,002)$, conversação e desenvoltura social $(p=0,008)$ e auto-exposição a desconhecidos e situações novas $(p=0,008)$.

Tabela 1

Dados estatísticos de mudanças verificadas nos escores fatoriais do Inventário de Habilidades Sociais no grupo experimental entre os periodos antes e depois da intervenção

\begin{tabular}{|c|c|c|c|c|c|c|c|}
\hline Fatores & & Média & $\begin{array}{l}\text { Desvio } \\
\text { padrão }\end{array}$ & $\begin{array}{c}\text { Erro } \\
\text { padrão }\end{array}$ & $t$ & $g l$ & $p$ \\
\hline \multirow{2}{*}{ Fator 1 - Enfrentamento e auto-afirmação com risco } & Pré & 0,166 & 0,839 & 0,203 & \multirow{2}{*}{11,374} & \multirow{2}{*}{16} & \multirow{2}{*}{0,002} \\
\hline & Pós & 0,194 & 1,230 & 0,298 & & & \\
\hline \multirow{2}{*}{ Fator 2 - Auto-afirmação na expressão de sentimentos positivos } & Pré & 0,040 & 0,690 & 0,158 & \multirow{2}{*}{0,886} & \multirow{2}{*}{18} & \multirow{2}{*}{0,387} \\
\hline & Pós & 0,364 & 1,224 & 0,281 & & & \\
\hline \multirow{2}{*}{ Fator 3 - Conversação e desenvoltura social } & Pré & 0,147 & 0,972 & 0,229 & \multirow{2}{*}{8,226} & \multirow{2}{*}{17} & \multirow{2}{*}{0,008} \\
\hline & Pós & 0,224 & 1,102 & 0,260 & & & \\
\hline \multirow{2}{*}{ Fator 4 - Auto-exposição a desconhecidos e situações novas } & Pré & 0,119 & 1,052 & 0,241 & \multirow{2}{*}{$-7,281$} & \multirow{2}{*}{18} & \multirow{2}{*}{0,008} \\
\hline & Pós & 0,210 & 1,022 & 0,234 & & & \\
\hline \multirow{2}{*}{ Fator 5 - Autocontrole da agressividade em situações aversivas } & Pré & 0,003 & 0,963 & 0,221 & \multirow{2}{*}{$-1,232$} & \multirow{2}{*}{18} & \multirow{2}{*}{0,234} \\
\hline & Pós & $-0,460$ & 1,079 & 0,247 & & & \\
\hline
\end{tabular}

Já os dados estatísticos referentes às mudanças verificadas no escore total do IHS no grupo experimental, entre os períodos antes e depois do PHSI, mostrou um aumento significativo $(t=-2,82, g l=15, p=0,01)$ na média do grupo experimental.

No follow-up ocorreu um aumento estatisticamente significativo da primeira avaliação para a terceira, entre os participantes do grupo experimental, nos seguintes fatores do IHS: enfrentamento e auto-afirmação com risco $(t=6,82, g l=18, p=0,003)$, conversação e desenvoltura social $(t=9,85$, $g l=18, p=0,001)$ e auto-exposição a desconhecidos e situações novas $(t=7,91, g l=18, p=0,042)$. Entretanto, não ocorreu mudança significativa em nenhum dos fatores do IHS da segunda avaliação para a terceira, indicando que os resultados se mantiveram. 
A partir da análise intragrupos, é possível observar que o PHSI foi eficaz em aumentar significativamente as respostas dos participantes experimentais no fator enfrentamento e auto-afirmação com risco; no fator conversação e desenvoltura social e no fator auto-exposição a desconhecidos e situações novas e esse aumento se manteve estável após um follow-up de 30 dias.

A comparação entre os grupos, controle e experimental, antes da intervenção, a partir do teste qui-quadrado McNemar, mostrou que os dois grupos, experimental e controle, eram equivalentes no que se refere ao desempenho verbal diante das sete situações de jogos de papéis. Já a comparação dos resultados entre grupos, na fase pós-intervenção, revelou que os participantes do grupo experimental apresentaram resultados superiores aos do grupo controle, nas sete situações de jogos de papéis, como mostra a Tabela 2.

\section{Tabela 2}

Comparação entre as respostas sociais inadequadas, respostas sociais parcialmente adequadas e respostas sociais adequadas dos grupos controle e experimental nas sete situações de jogos de papéis na fase pós-intervenção

\begin{tabular}{|c|c|c|c|c|c|c|}
\hline Situações sociais & Grupos & $\begin{array}{c}\text { Respostas } \\
\text { sociais } \\
\text { inadequadas }\end{array}$ & $\begin{array}{l}\text { Respostas } \\
\text { sociais } \\
\text { parcialmente } \\
\text { adequadas }\end{array}$ & $\begin{array}{l}\text { Respostas } \\
\text { sociais } \\
\text { adequadas }\end{array}$ & $\chi^{2}$ & $\mathrm{gl}$ \\
\hline \multirow{2}{*}{ 1. Iniciar Conversação } & Grupo Controle & $65 \%$ & $20 \%$ & $15 \%$ & \multirow{2}{*}{8,917} & \multirow{2}{*}{2} \\
\hline & Grupo Experimental & $10 \%$ & $50 \%$ & $40 \%$ & & \\
\hline \multirow{2}{*}{ 2. Recusar Pedido } & Grupo Controle & $65 \%$ & $5 \%$ & $30 \%$ & \multirow{2}{*}{10,003} & \multirow{2}{*}{2} \\
\hline & Grupo Experimental & $45 \%$ & $5 \%$ & $50 \%$ & & \\
\hline \multirow{2}{*}{ 3. Expressar Opinião Pessoal } & Grupo Controle & $90 \%$ & $10 \%$ & $0 \%$ & \multirow{2}{*}{2,131} & \multirow{2}{*}{2} \\
\hline & Grupo Experimental & $75 \%$ & $10 \%$ & $15 \%$ & & \\
\hline \multirow{2}{*}{ 4. Cobrar Dívida } & Grupo Controle & $60 \%$ & $15 \%$ & $25 \%$ & \multirow{2}{*}{10,628} & \multirow{2}{*}{2} \\
\hline & Grupo Experimental & $30 \%$ & $30 \%$ & $40 \%$ & & \\
\hline \multirow{2}{*}{$\begin{array}{l}\text { 5. Defender os próprios direitos em situações nas } \\
\text { quais são oferecidos serviços insatisfatórios }\end{array}$} & Grupo Controle & $60 \%$ & $15 \%$ & $25 \%$ & \multirow[b]{2}{*}{9,222} & \multirow[b]{2}{*}{2} \\
\hline & Grupo Experimental & $30 \%$ & $30 \%$ & $40 \%$ & & \\
\hline \multirow{2}{*}{ 6. Lidar com pessoas com atitudes grosseiras } & Grupo Controle & $65 \%$ & $30 \%$ & $5 \%$ & \multirow{2}{*}{4,721} & \multirow{2}{*}{2} \\
\hline & Grupo Experimental & $55 \%$ & $30 \%$ & $15 \%$ & & \\
\hline \multirow{2}{*}{ 7. Fazer pedido com conflito de interesses } & Grupo Controle & $70 \%$ & $30 \%$ & $0 \%$ & \multirow{2}{*}{11,022} & \multirow{2}{*}{2} \\
\hline & Grupo Experimental & $55 \%$ & $35 \%$ & $10 \%$ & & \\
\hline
\end{tabular}

$\mathrm{Na}$ fase pós-intervenção, foram encontradas diferenças significativas nas situações de iniciar conversação $(p=0,002)$, de recusar pedido $(p=0,001)$, de cobrar dívida $(p=0,001)$, de defender os próprios direitos $(p=0,002)$, de lidar com pessoas com atitudes grosseiras $(p=0,04)$ e de fazer pedido com conflito de interesses $(p=0,001)$. A seguir, será apresentada a análise intragrupos nas sete situações de jogos de papéis.

A análise dos dados, obtida a partir da avaliação dos juízes, revelou que em relação ao grupo controle, não foram encontradas diferenças significativas em nenhuma das sete situações de jogos de papéis, entre os períodos antes e depois do PHSI. Já em relação ao grupo experimental, foram encontradas diferenças significativas, entre os períodos antes e depois do PHSI, em quatro das sete situações de jogos de papéis, conforme pode ser visualizado na Tabela 3. 
Tabela 3

Dados estatísticos de mudanças verificadas nas sete situações de jogos de papéis, entre os periodos antes e depois da intervenção, no grupo experimental

\begin{tabular}{|c|c|c|c|c|}
\hline \multirow{3}{*}{$\begin{array}{l}\text { Situações Sociais } \\
\text { 1. Iniciar conversação }\end{array}$} & \multicolumn{3}{|c|}{ Média Rank } & \multirow{3}{*}{$\begin{array}{l}-3,540 \\
<0,001\end{array}$} \\
\hline & Pré & 0,000 & Z & \\
\hline & Pós & 8,000 & Asymp. Sig. & \\
\hline \multirow{2}{*}{ 2. Recusar pedido } & Pré & 1,500 & Z & $-2,200$ \\
\hline & Pós & 4,420 & Asymp. Sig. & 0,030 \\
\hline \multirow{2}{*}{ 3. Expressar opinião pessoal } & Pré & 5,130 & $\mathrm{Z}$ & $-0,250$ \\
\hline & Pós & 4,900 & Asymp. Sig. & 0,800 \\
\hline \multirow{2}{*}{ 4. Cobrar dívida } & Pré & 0,000 & $\mathrm{Z}$ & $-2,070$ \\
\hline & Pós & 3,000 & Asymp. Sig. & 0,040 \\
\hline \multirow{2}{*}{$\begin{array}{l}\text { 5. Defender os próprios direitos em situações nas quais são } \\
\text { oferecidos serviços insatisfatórios }\end{array}$} & Pré & 3,000 & $\mathrm{Z}$ & $-2,165$ \\
\hline & Pós & 4,710 & Asymp. Sig. & 0,030 \\
\hline \multirow{2}{*}{ 6. Lidar com pessoas com atitudes grosseiras } & Pré & 5,500 & $\mathrm{Z}$ & $-1,604$ \\
\hline & Pós & 6,200 & Asymp. Sig. & 0,109 \\
\hline \multirow{2}{*}{ 7. Fazer pedido com conflito de interesses } & Pré & 4,000 & $\mathrm{Z}$ & $-1,890$ \\
\hline & Pós & 4,000 & Asymp. Sig. & 0,059 \\
\hline
\end{tabular}

A partir da Tabela 3, observa-se que os participantes do grupo experimental apresentaram melhora significativa nas situações de iniciar conversação $(p=0,00)$; de recusar pedido $(p=0,03)$; de cobrar dívida $(p=0,04)$ e de defender os próprios direitos $(p=0,03)$.

Em relação ao follow-up ocorreu um aumento significativo no grupo experimental, segundo o teste qui-quadrado, da primeira para a terceira avaliação nas situações de: iniciar conversação $(p=0,004)$, recusar pedido $(p=0,01)$, cobrar dívida $(p=0,04)$ e defender os próprios direitos $(p=0,001)$. No entanto, não ocorreu mudança significativa em nenhuma das situações de jogos de papéis da segunda para a terceira avaliação. Desta forma, é possível afirmar que as respostas da maioria dos participantes do grupo experimental melhoraram da primeira para a segunda avaliação e se mantiveram estáveis da segunda para a terceira avaliação.

\section{Discussão}

Com base nos dados desse estudo, o PHSI mostrou-se significativamente eficaz no desempenho verbal dos participantes que receberam a intervenção, em relação ao grupo controle, nas situações que envolvem conflito de interesses (e.g., recusar pedidos, cobrar dívidas, defender os próprios direitos em situações nas quais são oferecidos serviços insatisfatórios, lidar com pessoas com atitudes grosseiras e fazer pedido com conflito de interesses). Uma vez que essas habilidades assertivas têm se mostrado deficitárias em idosos e que a necessidade de treinamento destas tem sido ressaltada na literatura (Carneiro \& Falcone, 2004; Cavalcanti, 1995), o PHSI pode se constituir como um trabalho de intervenção útil às demandas dessa população.

Ao desenvolver habilidades assertivas, o idoso poderá expressar as suas necessidades de forma adequada ao contexto, promovendo elevação da autoestima e evitando a manifestação de comportamento hostil. De acordo com Vila (2005), a expressão assertiva de um incômodo 
promove redução de perdas, tanto para o indivíduo quanto para o interlocutor, além de possibilitar bem-estar emocional e fornecer modelo de controle de reações emocionais indesejáveis.

Análises intragrupos realizadas com os escores do IHS também confirmaram a eficácia do PHSI para a capacidade de lidar com situações interpessoais que demandam a afirmação e defesa de direitos e auto-estima, com risco potencial de reação indesejável por parte do interlocutor (possibilidade de rejeição, de réplica ou de oposição). Isso indica que os participantes do grupo experimental desenvolveram a habilidade assertiva para lidar com situações como, por exemplo, devolver à loja uma mercadoria defeituosa; discordar do grupo; lidar com críticas injustas; cobrar dívida de amigo.

Com relação às habilidades de conversação e desenvoltura social, que retratam a capacidade de lidar com situações sociais neutras (e.g., manter e encerrar conversação, abordar pessoas que ocupam posição de autoridade, pedir favor a colegas e desconhecidos, recusar pedidos abusivos, fazer apresentações ou palestras a um público desconhecido e fazer perguntas a pessoas desconhecidas), pôde-se observar uma melhora significativa entre os participantes experimentais após o PHSI. Del Prette e Del Prette (2001) enfatizam a importância das habilidades sociais de conversação no repertório comportamental, uma vez que estas favorecem o traquejo social. Do ponto de vista de Bolsoni-Silva e Marturano (2002), essa classe de habilidades auxilia no desenvolvimento e manutenção de conversas amistosas e triviais.

Entretanto, os resultados a partir do IHS não revelaram mudanças significativas nos Fatores de autoafirmação na expressão de sentimento positivo (e.g., agradecer elogios; fazer elogios; expressar sentimentos positivos) e do autocontrole da agressividade em situações aversivas (e.g., lidar com críticas ou chacotas). É importante levar em consideração que os participantes tanto do grupo controle como do grupo experimental são estudantes de uma Universidade para a Terceira Idade e, conforme propõe Cachioni (1998), as Universidades da Terceira Idade constituem um espaço importante para a ampliação dos contatos interpessoais e a promoção das habilidades sociais.

Os resultados do Fator referente ao autocontrole da agressividade revelaram-se mais deficitários ao final do PHSI do que no início, mostrando, desta forma, uma resposta contrária à que era inicialmente esperada. Tal constatação está em consonância com os discutidos pela literatura (e.g., Magalhães \& Murta, 2003; Pacheco \& Rangé, 2006) os quais indicam que é possível que os participantes com estilo de comunicação não assertivo, ao entrarem em contato com instruções sobre defesa de direitos interpessoais e assertividade, passem a empregar o estilo de comunicação agressivo em suas relações para, posteriormente, expressarem a raiva e o desagrado de forma socialmente competente. Outra possibilidade para explicar essa diminuição de valores na segunda avaliação, em programa com população não-clínica, pode se relacionar, segundo Del Prette e Del Prette (2003), à ansiedade devido à evocação de situações de desempenho trazidas pelo inventário, muito mais acentuada na segunda avaliação do que na primeira, quando a preocupação com a competência era menor.

Um dado relevante para pesquisa envolvendo o desenvolvimento de habilidades sociais refere-se à escolha das medidas de avaliação. Verificou-se que, neste estudo, há necessidade da utilização de mais de um recurso de avaliação nos estudos de eficácia de intervenção, uma vez que esse procedimento supre as limitações de cada medida (Falcone, 1998; Magalhães \& Murta, 2003).

\section{Considerações finais}

Os resultados deste estudo fornecem subsídios para a implantação de programas de Desenvolvimento de Habilidades Sociais específicos para terceira idade. De acordo com Matos, 
Branco, Carvalhosa, Silva e Carvalhosa (2005), intervenções deste tipo indicam diminuir o encargo com pedidos exagerados de cuidados médicos e de apoio social que muitas vezes aparecem associados a situações de isolamento social e sentimento de solidão.

Como contribuição para estudos posteriores: o aumento do número de participantes da amostra, a realização do follow-up com os participantes do grupo controle, o uso de medidas tanto quantitativas como qualitativas, a avaliação da habilidade empática. Para melhorar a eficácia do PHSI algumas estratégias sugeridas são: o aumento do número de encontros, fortalecendo a aprendizagem; a criação de mais exercícios, visando desenvolver a competência social nas situações de expressar opinião pessoal, agradecer elogios, fazer elogios, expressar sentimentos positivos, lidar com críticas e lidar com chacotas.

Conclui-se que o PHSI mostrou-se eficaz em aumentar o repertório de habilidades sociais em idosos. Esses resultados ratificam estudos anteriores que sustentam que as habilidades sociais podem ser aprendidas em qualquer fase do desenvolvimento humano e que podem melhorar a partir de programas de intervenção adequados (Braz \& Del Prette, 2011; Isquick, 1981). Por outro lado, considerando alguns limites identificados neste estudo, recomendam-se novas investigações nessa linha para verificar a eficácia desse tipo de intervenção.

\section{Referências}

Almeida, O. P., \& Almeida, S. A. (1999). Confiabilidade da versão brasileira da escala de depressão em geriatria (GDS) versão reduzida. Arq Neuro-Psiquiatr, 57, 421-426.

Baltes, P. B. (1995). Prefácio. In A. L. Neri (Ed.), Psicologia do envelhecimento (pp. 9-12). Campinas: Papirus.

Barrett-Lennard, G. T. (1993). The phases and focus of empathy. British Journal of Medical Psychology, 66, 3-14. doi: 10.1111/j.2044-8341.1993.tb01722.x

Bolsoni-Silva, A. T., \& Marturano, E. M. (2002). Práticas educativas e problemas de comportamento: Uma análise à luz das habilidades sociais. Estudos de Psicologia (Natal), 7, 227-235.

Braz, A. C., \& Del Prette, Z. A. P. (2011). Programa de habilidades sociais assertivas para idosos. In A. Del Prette \& Z. A. P. Del Prette (Orgs.), Habilidades sociais: Intervenções efetivas em grupo (pp. 231-260). São Paulo: Casa do Psicólogo.

Cachioni, M. (1998). Envelhecimento bem-sucedido e participação numa universidade para a terceira idade: A experiência dos alunos da Universidade São Francisco. Dissertação de mestrado, UNICAMP, Campinas.

Capitanini, M. E. S. (2000). Solidão na velhice: Realidade ou mito?. In A. L. Neri \& S. A. Freire (Eds.), E por falar em boa velhice (pp. 69-89). Campinas: Papirus.

Carneiro, R. S., \& Falcone, E. O. (2004). Um estudo das capacidades e deficiências em habilidades sociais na terceira idade. Psicologia em Estudo, 9, 119-126.

Carneiro, R. S., Falcone, E., Clark, C., Del Prette, Z., \& Del Prette, A. (2007). Qualidade de vida, apoio social e depressão em idosos: Relação com habilidades sociais. Psicologia: Reflexão e Crítica, 20, 229-237.

Cavalcanti, M. B. (1995). Idosos. In B. Rangé (Ed.), Psicoterapia comportamental (pp. 149-158). Rio de Janeiro: Psy.

Del Prette, A., \& Del Prette, Z. A. P. (2003). No contexto da travessia para o ambiente de trabalho: Treinamento de habilidades sociais com universitários. Estudos de Psicologia, 8, 413-420.

Del Prette, A., \& Del Prette, Z. A. P. (2011). Enfoques e modelos do treinamento de habilidades sociais. In A. Del Prette \& Z. A. P. Del Prette (Eds.), Habilidades sociais: Intervenções efetivas em grupo (pp. 19-56). São Paulo: Casa do Psicólogo. 
Del Prette, Z. A. P., \& Del Prette, A. (2001). Inventário de habilidades sociais: Manual de aplicação, apuração e interpretação. São Paulo: Casa do Psicólogo Livraria e Editora Ltda.

Del Prette, Z. A. P., \& Del Prette, A. (2008). Um sistema de categorias de habilidades sociais educativas. Paidéia (Ribeirão Preto), 18, 517-530.

Falcone, E. M. O. (1998). A avaliação de um programa de treinamento da empatia. Tese de doutorado, Universidade de São Paulo, São Paulo.

Falcone, E. M. O. (2001). Uma proposta de um sistema de classificação das habilidades sociais. In H. J. Guilhardi, M. B. B. P. Madi, P. P. Queiroz, \& M. C. Scoz (Eds.), Sobre comportamento e cognição: Expondo a variabilidade (pp. 195-209). Santo André: SET Editora.

Falcone, E. M. O., \& Ramos, D. M. (2005). A atribuição como componente cognitivo das habilidades sociais e seu impacto na satisfação conjugal. In H. J. Guilhardi \& N. C. Aguirre (Orgs.), Sobre comportamento e cognição: Expondo a variabilidade (pp. 182-191). Santo André: ESETec Editores Associados.

Falcone, E. M. O., Carneiro, R. S., Chicayban, L. M., Oliveira, M. G. S., Pedrozo, A. L. B., \& Ferreira, M. C. (2001). A construção de um sistema de classificação das habilidades sociais [Resumo]. In Congresso Iberoamericano de Psicologia Clínica e da Saúde (Org.), Avanços recentes em psicologia clínica e da saúde. Resumo do II Congresso Iberoamericano de Psicologia Clínica e da Saúde (p. 371). Guarujá: APICSA.

Freire, S. A., \& Sommerhalder, C. (2000). Envelhecer nos tempos modernos. In A. L. Neri \& S. A. Freire (Eds.), E por falar em boa velhice (pp. 125-135). Campinas: Papirus.

García-Vera, M. P., Sanz, J., \& Gil, F. (1998). Entrenamientos en habilidades sociales. In F. Gil \& J. M. León (Eds.), Habilidades sociales: Teoría, investigación e intervención (pp. 63-93). Madrid: Editorial Síntesis.

Goleman, D. (2006). Inteligência social: O poder das relações humanas (Trad. Ana Beatriz Rodrigues). Rio de Janeiro: Elsevier.

Herculano-Houzel, S. (2007). Fique de bem com seu cérebro: Guia prático para o bem-estar em 15 passos. Rio de Janeiro: Sextante.

Isquick, M. F. (1981). Training older people in empathy: Effects on empathy, attitudes, and self-exploration. Int $J$ Aging and Human Development, 13, 1-14.

Joia, L. C., Ruiz, T., \& Donalisio, M. R. (2007). Condições associadas ao grau de satisfação com a vida entre a população de idosos. Revista de Saúde Pública, 41, 131-138.

Katz, L., \& Rubin, M. (2000). Mantenha o seu cérebro vivo. São Paulo: Sextante.

Lange, A., \& Jakubowski, P. (1976). Responsible assertive behavior. Illionis: Ed. Research Press.

Magalhães, P. P., \& Murta, S. G. (2003). Treinamento de habilidades sociais em estudantes de psicologia: Um estudo pré-experimental. Temas em Psicologia da SBP, 11, 28-37.

Matos, M. G. de, Branco, J. D., Carvalhosa, S. F., Silva, M. N., \& Carvalhosa, J. (2005). Promoção de competências pessoais e sociais nos idosos: Programa de intervenção na comunidade. Revista Brasileira de Terapias Cognitivas, 1, 95-102.

Matsukura, T. S., Marturano, E. M., \& Oishi, J. O. (2002). O Questionário de Suporte Social (SSQ): Estudos da adaptação para o português. Revista Latino-Americana de Enfermagem, 10, 675-681.

Neri, A. L. (2004). Contribuições da psicologia ao estudo e à intervenção no campo da velhice. Revista Brasileira de Ciências do Envelhecimento Humano, Passo Fundo, 1, 69-80.

Pacheco, P., \& Rangé, B. (2006). Desenvolvimento de habilidades sociais em graduandos de psicologia. In M. Bandeira, Z. Del Prette, \& A. Del Prette (Eds.), Estudos sobre habilidades sociais e relacionamento interpessoal (pp. 199-216). São Paulo: Casa do Psicólogo.

Ramos, M. P. (2002). Apoio social e saúde entre idosos. Sociologias, 7, 156-175. 
Ramos, M. (2007). Os sintomas depressivos e as relações sociais na terceira idade. Revista do Departamento de Psicologia-UFF, 19, 397-410.

Resende, M. C. de, Bones, V. M., Souza, I. S., \& Guimarães, N. K. (2006). Rede de relações sociais e satisfação com a vida de adultos e idosos. Psicol Am Lat, 5, 1-15.

Veras, R. P. (2002). O anacronismo dos modelos assistenciais na área da saúde: Mudar e inovar, desafios para o setor público e o privado. In R. P. Veras (Org.), Terceira idade: Gestão contemporânea em saúde (pp. 163-185). Rio de Janeiro: Relume Dumará.

Vila, E. M. (2005). Treinamento de habilidades sociais em grupo com professores de crianças com dificuldades de aprendizagem: Uma análise sobre procedimentos e efeitos da intervenção. Dissertação de mestrado, Universidade Federal de São Carlos, São Paulo, Brasil.

Vila, E. M., Gongora, M. A. N., \& Silveira, J. M. (2003). Ensinando repertório alternativo para clientes que apresentam padrões comportamentais passivo e hostil. In C. G. de Almeida (Org.), Intervenções em grupos: Estratégias psicológicas para a melhoria da qualidade de vida (pp. 59-81). Campinas: Papirus.

Evidence in the literature point to the importance of quality in social relationships, but do not provide enough data that indicates how to assist the elderly to become socially competent. This study aims to evaluate the effectiveness of a Program to Promotion Social Skills for the Elderly. The research had the participation of 40 students at the University of the Third Age. The experimental group included 20 people with ages between $62-83$ years $(M=69.4 ; S D=6.05)$ and the control group had 20 elderly with ages between $60-74$ years $(M=67.1 ; S D=4.61)$. Social skills were checked before and after intervention with follow-up through a measure of self-report and performance through role play situations. The results showed significant improvement in social performance of the participants in the experimental group compared to the control group, in contexts involving conflict of interest. Intragroup analysis also confirmed an improvement in the capacity to handle interpersonal situations that require the affirmation and defense of rights. These results find that the created program promoted assertive skills, previously identified in the literature as deficient in the elderly people.

Key words: Elderly, Program to promotion social skills, Social performance. 
